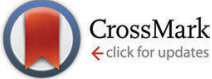

Cite this: Phys. Chem. Chem. Phys., 2016, 18, 22031

Received 31st March 2016 Accepted 20th July 2016

DOI: $10.1039 / c 6 c p 02131 j$

www.rsc.org/pccp

\title{
Oxygen exchange at gas/oxide interfaces: how the apparent activation energy of the surface exchange coefficient depends on the kinetic regime
}

\author{
Peter Fielitz* and Günter Borchardt
}

\begin{abstract}
In the dedicated literature the oxygen surface exchange coefficient $K_{\mathrm{O}}$ and the equilibrium oxygen exchange rate $\mathrm{i}_{\mathrm{O}}^{0}$ are considered to be directly proportional to each other regardless of the experimental circumstances. Recent experimental observations, however, contradict the consequences of this assumption. Most surprising is the finding that the apparent activation energy of $K_{\mathrm{O}}$ depends dramatically on the kinetic regime in which it has been determined, i.e. surface exchange controlled vs. mixed or diffusion controlled. This work demonstrates how the diffusion boundary condition at the gas/solid interface inevitably entails a correlation between the oxygen surface exchange coefficient $K_{\circ}$ and the oxygen selfdiffusion coefficient $D_{O}$ in the bulk ("on top" of the correlation between $K_{\mathrm{O}}$ and $\Re_{0}^{0}$ for the pure surface exchange regime). The model can thus quantitatively explain the range of apparent activation energies measured in the different regimes: in the surface exchange regime the apparent activation energy only contains the contribution of the equilibrium exchange rate, whereas in the mixed or in the diffusion controlled regime the contribution of the oxygen self-diffusivity has also to be taken into account, which may yield significantly higher apparent activation energies and simultaneously quantifies the correlation $K_{\mathrm{O}} \propto D_{\mathrm{O}}^{1 / 2}$ observed for a large number of oxides in the mixed or diffusion controlled regime, respectively.
\end{abstract}

As early as 1996 Kilner et $a l^{1}{ }^{1}$ showed that there is, for a large number of different materials, a quite obvious correlation $K_{\mathrm{O}} \propto D_{\mathrm{O}}{ }^{1 / 2}$ between the oxygen surface exchange coefficient $K_{\mathrm{O}}$ and the oxygen diffusion coefficient $D_{\mathrm{O}}$ (over almost $10(!)$ orders of magnitude for $D_{\mathrm{O}}$ ) for a number of perovskite compositions (with predominant electronic conductivity). Despite this empirical evidence it is assumed until to date, ${ }^{2-4}$ however, that $K_{\mathrm{O}}$ and $D_{\mathrm{O}}$ are independent parameters, each of which reflects an independent microscopic mechanism. A major reason for this picture can be identified in the methodology of standard oxygen exchange experiments: ${ }^{5}$ Here the oxygen

Technische Universität Clausthal, Institut für Metallurgie, Robert-Koch-Str. 42, D-38678 Clausthal-Zellerfeld, Germany.E-mail: peter.fielitz@tu-clausthal.de flux $j_{\mathrm{O}}^{K}$ through a surface element subject to a concentration difference $\Delta c_{\mathrm{O}}$ is expressed as follows

$$
j_{\mathrm{O}}^{K}(0)=-K_{\mathrm{O}} \Delta c_{\mathrm{O}}(0)
$$

The phenomenological eqn (1) defines $K_{\mathrm{O}}$ without recurring to any microscopic mechanism. As eqn (1) is only valid for surface reactions at $x=0$ it seemed plausible that $K_{\mathrm{O}}$ depends exclusively on the defect chemistry of the surface. In 1998 Maier $^{6}$ proposed a relation between $K_{\mathrm{O}}$ and the equilibrium oxygen exchange rate $\mathfrak{R}_{\mathrm{O}}^{0}$ at the gas/solid interface (s. Maier, ${ }^{6}$ p. 222, eqn (149))

$$
\frac{K_{\mathrm{O}}}{\Delta x}=\frac{\Re_{\mathrm{O}}^{0}}{c_{\mathrm{O}}(0)} w_{\mathrm{O}}(0) \quad \text { with }\left.\quad w_{\mathrm{O}}(0) \equiv \frac{c_{\mathrm{O}}(0)}{R T} \frac{\partial \mu_{\mathrm{O}}}{\partial c_{\mathrm{O}}}\right|_{x=0}
$$

where $c_{\mathrm{O}}(0)$ is the oxygen equilibrium concentration near the surface, $w_{\mathrm{O}}(0)$ is the thermodynamic factor near the surface, $R$ and $T$ have the usual meaning, and $\mu_{\mathrm{O}}$ is the chemical potential of oxygen. $\mathfrak{R}_{\mathrm{O}}^{0}$ is the oxygen exchange rate of the rate determining step in a consecutive reaction sequence. ${ }^{7,8}$ Without violating Maier's universally valid original approach, in the literature $e^{4,9-14}$ also an effective oxygen exchange rate $\left(1 / \Re_{\mathrm{O}}^{0}=\sum_{i=1}^{n} 1 / \Re_{i}\right.$, $n=$ number of consecutive reactions) is used to describe socalled consecutive "heterogeneous" reactions. The quantitative measure of the parameter $\Delta x$ (with the dimension of a length) having been left undefined, Maier's suggestion essentially means that $K_{\mathrm{O}}$ is proportional to $\mathfrak{R}_{\mathrm{O}}^{0}{ }^{15}$

For the oxygen isotope exchange the thermodynamic factor is unity and eqn (2) yields $\tilde{K}_{\mathrm{O}}=\mathfrak{w}(0) K_{\mathrm{O}}{ }^{*}$. (Throughout the text an asterisk characterizes a parameter determined in an isotope exchange experiment, whereas a tilde refers to a parameter determined in a chemical exchange experiment.) From $\tilde{D}_{\mathrm{O}}=$ $w(0) D_{\mathrm{O}}=w(0) D_{\mathrm{O}} * / f_{\mathrm{O}}$, where $D_{\mathrm{O}}$ is the self-diffusion coefficient and $D_{\mathrm{O}}{ }^{*}$ is the tracer diffusion coefficient of oxygen, respectively, follows with the correlation factor $f_{\mathrm{O}} \simeq 1$ a simple theoretical correlation. ${ }^{15}$

$$
\frac{K_{\mathrm{O}}{ }^{*}}{D_{\mathrm{O}}{ }^{*}} \cong \frac{\tilde{K}_{\mathrm{O}}}{\tilde{D}_{\mathrm{O}}}
$$


In 2006 De Souza $^{7}$ explicitly pointed out that there is a generally observed discrepancy between experimentally determined $K_{\mathrm{O}} / D_{\mathrm{O}}$ ratios and the theoretically expected ratios according to eqn (3). Instead of fulfilling eqn (3) the experimental data yield $K_{\mathrm{O}}{ }^{*} / D_{\mathrm{O}}{ }^{*}>\tilde{K}_{\mathrm{O}} / \tilde{D}_{\mathrm{O}}$. This was a first significant experimental indication that eqn (2) might not fully take into account all aspects of the problem.

Armstrong et al. $^{12,13}$ used in 2011 and 2013 a novel experimental approach, called isothermal isotope exchange (IIE), to extract accurate $K_{\mathrm{O}}{ }^{*}$ values in a surface exchange controlled regime. This technique relies on the exchange of isotopically labelled oxygen for lattice oxygen in a similar way to isotope exchange depth profiling (IEDP) with secondary ion mass spectrometry (SIMS), but is conducted in situ on powder samples (particle size 200-300 $\mathrm{nm}$ ). In this way the exchange kinetics can be expected to be clearly in the surface exchange regime. Very unexpectedly Armstrong et al. ${ }^{12,13}$ found apparent activation energies of $K_{\mathrm{O}}{ }^{*}$ with negative (!) or very small positive values for different lanthanum based MIEC perovskite oxides like LC, LF, LSM, LSF, LSC or LSCF, but also for the standard oxygen ion conductors YSZ and GDC, which have fluorite structure. The reported range of apparent activation energies of the oxygen tracer surface exchange coefficient is $-97 \mathrm{~kJ} \mathrm{~mol}^{-1}$ to $+12 \mathrm{~kJ} \mathrm{~mol}^{-1}$ and is in strong contrast to the reported range from $+60 \mathrm{~kJ} \mathrm{~mol}^{-1}$ to $+333 \mathrm{~kJ} \mathrm{~mol}^{-1}$ measured by conventional methods generally using macroscopic sample geometries, i.e. IEDP/SIMS and electrical conductivity relaxation (ECR) (s. Armstrong et al., ${ }^{13}$ Table 5), but also measured by other powder-based methods like pulsed isotope exchange (PIE) ${ }^{9-11}$ and long-term gas phase exchange/analysis GPE/GPA.4,14,16 These experimental results suggest that the measured oxygen surface exchange coefficient $K_{\mathrm{O}}$, if measured in the mixed regime and in the diffusion controlled regime, may depend not only on the equilibrium oxygen exchange rate $\mathfrak{R}_{\mathrm{O}}^{0}$ at the gas/solid interface but also on the oxygen diffusion coefficient $D_{\mathrm{O}}$ in the bulk. Such a behaviour would clearly contradict eqn (2) which postulates that $K_{\mathrm{O}}$ depends on $\mathfrak{R}_{\mathrm{O}}^{0}$ only in all kinetic regimes.

In 2015 Knoblauch et al. ${ }^{17}$ performed thermogravimetric relaxation experiments on sintered $\mathrm{CeO}_{2}$ pellets $(1 \mathrm{~mm}$ thickness, $93 \%$ of theoretical density) with a grain size of about $20 \mu \mathrm{m}$. Because of the strongly enhanced chemical diffusivity ${ }^{18}$ during the oxygen reduction step of nominally undoped $\mathrm{CeO}_{2}$ Knoblauch et $a{ }^{17}{ }^{17}$ concluded that their reduction experiments had been performed in the surface exchange regime. They also reported an apparent negative activation energy of $-64 \mathrm{~kJ} \mathrm{~mol}^{-1}$ for $\tilde{K}_{\mathrm{O}}$. That is, the work of Knoblauch et al. ${ }^{17}$ showed that the apparent activation energy of the chemical oxygen surface exchange coefficient $\tilde{K}_{\mathrm{O}}$ can also have negative values if it is measured in the surface exchange controlled regime.

The experimental contradictions to the general validity (i.e. in all kinetic regimes) of eqn (2) necessitate a rediscussion of the meaning of the parameter $K_{\mathrm{O}}$. In order to facilitate a fundamental theoretical discussion the experimental situation of the oxygen exchange experiments is idealized as far as possible. In analogy to Maier ${ }^{6}$ jump relaxation effects, space charge effects, structural changes and trapping effects are considered secondary.

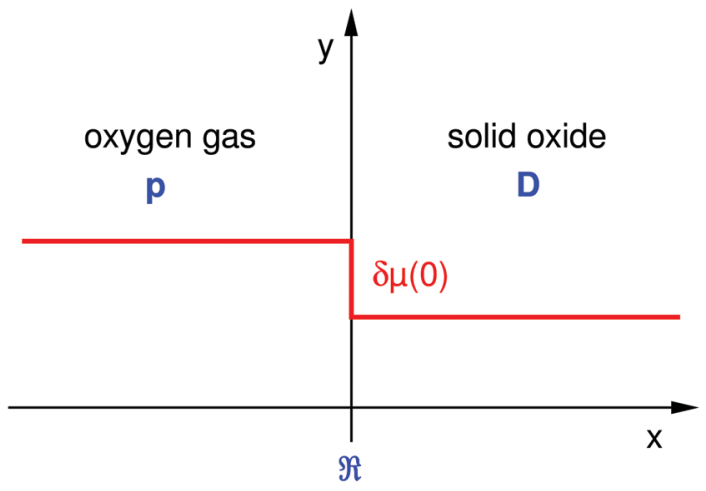

gas/oxide interface

Fig. 1 Schematic representation of a gas/oxide interface of a homogeneous semi-infinite solid oxide. $D$ is the self-diffusion coefficient of oxygen ions $\left(D_{\circ}\right)$ in the solid oxide, $p$ is the oxygen partial gas pressure $\left(p_{\mathrm{O}_{2}}\right)$ in the oxygen gas phase and $\Re$ is the equilibrium oxygen exchange rate $\left(\mathfrak{R}_{\mathrm{O}}^{0}\right)$ at the interface. At $t=0$ a small step-like change $\delta \mu(0)$ of the chemical potential of oxygen $\left(\delta \mu_{\circ}(x=0)\right)$ is experimentally induced at the gas/oxide interface at $x=0$.

Likewise the mathematical treatment is simplified by considering a homogeneous semi-infinite solid oxide (s. Fig. 1). In this case one has to consider only one gas/oxide interface at $x=0$ where defect chemical reactions at the interface are quantified by the equilibrium oxygen exchange rate $\mathfrak{R}_{\mathrm{O}}^{0}$. The diffusion process of oxygen ions in the solid oxide is quantified by the oxygen self-diffusion coefficient $D_{\mathrm{O}}$. The oxygen partial pressure in the gas phase is given by $p_{\mathrm{O}_{2}}$. That is, the considered oxide is phenomenologically described by two independent material parameters

$$
\mathfrak{R}_{\mathrm{O}}^{0}=\mathfrak{R}_{\mathrm{O}}^{0}\left(T, p_{\mathrm{O}_{2}}\right), \quad D_{\mathrm{O}}=D_{\mathrm{O}}\left(T, p_{\mathrm{O}_{2}}\right)
$$

which are functions of the temperature $T$ and the oxygen partial pressure $p_{\mathrm{O}_{2}}$, which control (together with unintentionally introduced impurities and/or deliberately added dopants) the concentration of oxygen vacancies $\left[\mathrm{V}_{\mathrm{O}}^{\bullet \bullet}\right]$ or interstitials $\left[\mathrm{O}_{i}^{\prime \prime}\right]$ (and of the other ionic and electronic defects). The oxygen surface exchange coefficient $K_{\mathrm{O}}$, however, is a phenomenological parameter which, a priori, is not yet related to a specific microscopic process via eqn (1). In view of the experimental findings outlined above it can be tentatively suggested that, at least for IEDP/SIMS and ECR experiments, but most probably also for PIE ${ }^{9-11}$ and GPE/GPA ${ }^{4,14,16}$ experiments, $K_{\mathrm{O}}$ should depend on the two independent parameters $D_{\mathrm{O}}$ and $\mathfrak{R}_{\mathrm{O}}^{0}$ as follows

$$
K_{\mathrm{O}}=K_{\mathrm{O}}\left(D_{\mathrm{O}}, \mathfrak{R}_{\mathrm{O}}^{0}\right)
$$

In order to derive the hypothetical function (5) from fundamental principal relations it is appropriate to adopt Maier's ${ }^{6}$ notation and his very general procedure: the oxygen flux $j_{\mathrm{O}}^{K}(0)$ through the surface (at $x=0$ ) which is induced by a small change of the chemical potential of oxygen $\delta \mu_{\mathrm{O}}(0)$ becomes $^{6}$

$$
j_{\mathrm{O}}^{K}(0)=-\Lambda_{\mathrm{O}} \delta \mu_{\mathrm{O}}(0)=-\left.\Lambda_{\mathrm{O}} \frac{\partial \mu_{\mathrm{O}}}{\partial c_{\mathrm{O}}}\right|_{x=0} \delta c_{\mathrm{O}}(0)=-K_{\mathrm{O}} \delta c_{\mathrm{O}}(0)
$$


where $\Lambda_{\mathrm{O}}$ is the conductance and $\partial \mu_{\mathrm{O}} / \partial c_{\mathrm{O}}$ the reciprocal "chemical" capacity. It is important to keep in mind that eqn (6) is an approximation near chemical equilibrium. It is very instructive to recall Maier's comment on eqn (6): "It is worth noting that these relationships [eqn (6)] are quite general in that they do not preselect a certain mechanism."6 This statement implicitly comprises the fact that the parameter $K_{\mathrm{O}}$ principally can be the result of a combination of several mechanisms.

The diffusive oxygen flux ${ }^{19} j_{\mathrm{O}}^{D}(0)$ in the near-surface region of thickness $\delta x(0)$, which is driven by the gradient of the chemical potential of oxygen $\partial \mu_{\mathrm{O}} /\left.\partial x\right|_{x=0}$, becomes

$$
j_{\mathrm{O}}^{D}(0) \cdot \delta x(0)=-\left.\frac{D_{\mathrm{O}} c_{\mathrm{O}}(0)}{R T} \frac{\partial \mu_{\mathrm{O}}}{\partial x}\right|_{x=0} \delta x(0)=-\frac{D_{\mathrm{O}} c_{\mathrm{O}}(0)}{R T} \delta \mu_{\mathrm{O}}(0)
$$

where $D_{\mathrm{O}}$ is the oxygen self-diffusion coefficient and $c_{\mathrm{O}}(0)$ is the oxygen concentration (at the surface).

The first step in the derivation of the hypothetical function (5) is to study the correlation between $K_{\mathrm{O}}$ and $D_{\mathrm{O}}$ which principally results from the boundary condition at the gas/solid interface

$$
j_{\mathrm{O}}^{D}(0)=j_{\mathrm{O}}^{K}(0)
$$

which connects surface exchange and bulk transport and which therefore has to be respected when solving the respective diffusion equation. ${ }^{20}$ With eqn (6) and (7) the boundary condition eqn (8) yields

$$
\left(-\Lambda_{\mathrm{O}} \delta x(0)+\frac{D_{\mathrm{O}} c_{\mathrm{O}}(0)}{R T}\right) \delta \mu_{\mathrm{O}}(0)=0
$$

This means that for a moderate value of $\delta \mu_{\mathrm{o}}(0)$, i.e. a nearequilibrium situation at the surface, the (standard) boundary condition eqn (8) yields the relation $D_{\mathrm{O}} c_{\mathrm{O}}(0) / R T=\Lambda_{\mathrm{O}} \delta x(0)$. With the thermodynamic factor $w_{\mathrm{O}}(0)$ (s. eqn (2)) and $K_{\mathrm{O}}=$ $\Lambda_{\mathrm{O}} \partial \mu_{\mathrm{O}} /\left.\partial c_{\mathrm{O}}\right|_{x=0}$ (s. eqn (6)) one finally obtains the following general correlation between $K_{\mathrm{O}}$ and $D_{\mathrm{O}}$ which is valid in the near-equilibrium situation

$$
\delta x(0) \cdot K_{\mathrm{O}}=w_{\mathrm{O}}(0) \cdot D_{\mathrm{O}}
$$

In the second step the meaning of the parameter $\delta x(0)$ will be clarified. For an isotope exchange experiment $w_{\mathrm{O}}(0)=1$ is valid and eqn (10) simply becomes $\delta x^{*}(0)=D_{\mathrm{O}}^{*} / K_{\mathrm{O}}^{*}$, which is equivalent to the so-called characteristic length $l_{\mathrm{c}}^{0}$ used in the literature to roughly estimate in which kinetic regime a given oxygen exchange experiment is performed. Adopting this notation the subsequent discussion will be facilitated for the reader as eqn (10) now becomes

$$
\frac{l_{\mathrm{c}}^{0}}{w_{\mathrm{O}}(0)}=\frac{D_{\mathrm{O}}}{K_{\mathrm{O}}}
$$

Here the upper case index 0 means that $l_{\mathrm{c}}^{0}$ refers to a nearsurface layer (at $x=0$ ). Until now eqn (11) was only a formal mathematical rule to calculate $l_{\mathrm{c}}^{0}$ and results as such directly from the boundary condition eqn (8). The physical meaning of $l_{\mathrm{c}}^{0}$ will become evident if one defines with eqn (11) a characteristic time $t_{\mathrm{c}}^{0}$ (valid likewise for the near-surface region at $x=0$ ) as follows

$$
t_{\mathrm{c}}^{0} \equiv \frac{l_{\mathrm{c}}^{0}}{w_{\mathrm{O}}(0) K_{\mathrm{O}}}=\frac{D_{\mathrm{O}}}{K_{\mathrm{O}}^{2}}
$$

In Appendix $\mathrm{A}$ it is demonstrated that the characteristic time $t_{\mathrm{c}}^{0}$ physically corresponds to the relaxation time of a (sufficiently small) change in the oxygen potential $\delta \mu(0)$ at the surface. Eqn (12) leads to the following two relations

$$
l_{\mathrm{c}}^{0}=w_{\mathrm{O}}(0) \sqrt{D_{\mathrm{O}} t_{\mathrm{c}}^{0}} ; \quad K_{\mathrm{O}}=\sqrt{\frac{D_{\mathrm{O}}}{t_{\mathrm{c}}^{0}}}
$$

The characteristic length $l_{\mathrm{c}}^{0}$ is thus proportional to the diffusion length the oxygen ions cover in the near-surface region during the characteristic time $t_{\mathrm{c}}^{0}$. The term $K_{\mathrm{O}} / \Delta x$ (as formulated by Maier ${ }^{6}$ ) in eqn (2) can be considered equivalent to the inverse characteristic time $t_{\mathrm{c}}^{0}$. This relation suggests to postulate

$$
t_{\mathrm{c}}^{0} \stackrel{!}{=} \frac{c_{\mathrm{O}}(0)}{w(0) \cdot \Re_{\mathrm{O}}^{0}}
$$

Combining eqn (13) and (14) yields the missing correlation anticipated as "the hypothetical function" in eqn (5)

$$
K_{\mathrm{O}}^{h \rightarrow \infty}=\left(w_{\mathrm{O}}(0) \cdot D_{\mathrm{O}} \cdot \frac{\Re_{\mathrm{O}}^{0}}{c_{\mathrm{O}}(0)}\right)^{1 / 2}
$$

It should be recalled that eqn (15) is a consequence of a boundary condition, and hence, is valid in all experimental situations where the considered boundary condition (eqn (8)) is valid. The upper case index $h \rightarrow \infty$ refers to the fact that this boundary condition is often applicable to plate-like samples of large thickness $h$, that is, if $h \gg l_{\mathrm{c}}^{0}$.

For an oxygen isotope exchange experiment (with $w_{\mathrm{O}}(0)=1$ and $D_{\mathrm{O}}{ }^{*}=f_{\mathrm{O}} \cdot D_{\mathrm{O}}$ ) eqn (15) becomes (dropping the index $h \rightarrow \infty$ in order to simplify the notation in eqn (16)-(19))

$$
\left(K_{\mathrm{O}}^{*}\right)^{2}=D_{\mathrm{O}}{ }^{*} \cdot \frac{\Re_{\mathrm{O}}^{0}}{c_{\mathrm{O}}(0)}=f_{\mathrm{O}} \cdot D_{\mathrm{O}} \cdot \frac{\Re_{\mathrm{O}}^{0}}{c_{\mathrm{O}}(0)}
$$

Here $\left(K_{\mathrm{O}^{*}}, D_{\mathrm{O}}{ }^{*}\right)$ are data couples determined via an isotope exchange experiment. In a chemical oxygen exchange experiment (with $w(0) \gg 1^{21,22}$ and $\left.\tilde{D}_{\mathrm{O}}=w_{\mathrm{O}}(0) \cdot D_{\mathrm{O}}\right)$ the data couples $\left(\tilde{K}_{\mathrm{O}}, \tilde{D}_{\mathrm{O}}\right)$ are determined. In this case eqn (15) yields

$$
\left(\tilde{K}_{\mathrm{O}}\right)^{2}=\tilde{D}_{\mathrm{O}} \frac{\Re_{\mathrm{O}}^{0}}{c_{\mathrm{O}}(0)}=w_{\mathrm{O}}(0) \cdot \frac{D_{\mathrm{O}}{ }^{*}}{f_{\mathrm{O}}} \cdot \frac{\Re_{\mathrm{O}}^{0}}{c_{\mathrm{O}}(0)}=\frac{w_{\mathrm{O}}(0)}{f_{\mathrm{O}}}\left(K_{\mathrm{O}}{ }^{*}\right)^{2}
$$

that is, one has $\tilde{K}_{\mathrm{O}}=\left(w_{\mathrm{O}}(0) / f_{\mathrm{O}}\right)^{1 / 2} \cdot K_{\mathrm{O}}{ }^{*}$ in contrast to $\tilde{D}_{\mathrm{O}}=\left(w_{\mathrm{O}}(0) / f_{\mathrm{O}}\right) \cdot D_{\mathrm{O}}{ }^{*}$. Comparing $K_{\mathrm{O}} / D_{\mathrm{O}}$ ratios for the isotope exchange and for the chemical exchange experiment, respectively, one obtains from eqn (16) and (17) the following theoretical relations

$$
\frac{K_{\mathrm{O}}{ }^{*}}{D_{\mathrm{O}}{ }^{*}}=\sqrt{\frac{w_{\mathrm{O}}(0)}{f_{\mathrm{O}}}} \frac{\tilde{K}_{\mathrm{O}}}{\tilde{D}_{\mathrm{O}}}=\left(\frac{\Re_{\mathrm{O}}^{0}}{f_{\mathrm{O}} \cdot D_{\mathrm{O}} c_{\mathrm{O}}(0)}\right)^{1 / 2}
$$

Because of $f_{\mathrm{O}} \approx 1$ and $w_{\mathrm{O}}(0) \gg 1^{21,22}$ one immediately obtains $K_{\mathrm{O}}{ }^{*} / D_{\mathrm{O}}{ }^{*}>\tilde{K}_{\mathrm{O}} / \tilde{D}_{\mathrm{O}}$, which is in agreement with a large number of experimental results. ${ }^{7}$ Thus eqn (18) supports the postulate expressed in eqn (14). 
For more complicated sample geometries and correspondingly more complicated boundary conditions a more complicated correlation than eqn (15) is to be expected. The formal calculation of a characteristic length $l_{\mathrm{c}}^{0}$ by eqn (11) is mathematically always possible, but the result would mask the physical meaning of $l_{\mathrm{c}}^{0}$ which is explicitly expressed in eqn (13) only. Likewise the characteristic time $t_{\mathrm{c}}^{0}$ has been (almost completely) neglected in the materials science literature, which focuses practically exclusively on $K_{\mathrm{O}}$. It is, however, principally preferable for the discussion of experimental studies to consider the two characteristic parameters $t_{\mathrm{c}}^{0}$ and $l_{\mathrm{c}}^{0}$ compiled together with $K_{\mathrm{O}}$ in eqn (19)

$$
\begin{aligned}
& t_{\mathrm{c}}^{0}=\frac{c_{\mathrm{O}}(0)}{w(0) \cdot \Re_{\mathrm{O}}^{0}} ; \quad l_{\mathrm{c}}^{0}=w(0) \sqrt{D_{\mathrm{O}} t_{\mathrm{c}}^{0}} ; \quad K_{\mathrm{O}}=\sqrt{\frac{D_{\mathrm{O}}}{t_{\mathrm{c}}^{0}}} \\
& \text { if } h \gg l_{\mathrm{c}}^{0}
\end{aligned}
$$

where $h$ is the thickness of a plate-like sample. The set of eqn (19) shows that the condition $h \gg l_{\mathrm{c}}^{0}$ is realised if the oxygen selfdiffusion $D_{\mathrm{O}}$ is low and/or $\mathfrak{R}_{\mathrm{O}}^{0}$ is high and/or $w(0)$ is small. This means that oxygen exchange occurs practically only in a near-surface region of characteristic thickness $l_{\mathrm{c}}^{0}$ during the characteristic time $t_{\mathrm{c}}^{0}$. Consequently the oxygen exchange of the whole sample is hindered due to the slow oxygen self-diffusion in the bulk. This situation corresponds to the so-called diffusion controlled regime.

The very specific condition $h \approx l_{\mathrm{c}}^{0}$ means that within the characteristic time $t_{\mathrm{c}}^{0}$ practically all the oxygen of the sample is exchanged. This scenario is called mixed regime as the oxygen exchange rate at the surface $\left(\mathfrak{R}_{\mathrm{O}}^{0}\right)$ and the self-diffusion coefficient in the bulk $\left(D_{\mathrm{O}}\right)$ hinder the overall oxygen exchange simultaneously. Note that the condition $h \approx l_{\mathrm{c}}^{0}$ is more easily reached during chemical exchange (with $w(0) \gg 1$ ) than during isotope exchange (with $w(0)=1$ ) because during chemical exchange oxygen selfdiffusion is enhanced by the thermodynamic factor, $\tilde{D}_{\mathrm{O}}=w_{\mathrm{O}}(0) D_{\mathrm{O}}$.

The condition $h \ll l_{\mathrm{c}}^{0}$ means for a given value of $h$ that the oxygen exchange at the sample surface is sufficiently slow and/or oxygen bulk transport is sufficiently rapid. In this case the oxygen exchange of the whole sample is entirely dominated by the (apparent equilibrium) oxygen exchange rate $\mathfrak{R}_{\mathrm{O}}^{0}$ at the surface, and this corresponds to the so-called surface exchange regime. For a thin plate-like sample with thickness $h$ and a sphere with radius $r$ the solution of the diffusion equation for the oxygen exchange kinetics in the surface exchange regime can be approximated by

$$
\frac{c_{\mathrm{O}}(t)-c_{\mathrm{O}}(0)}{c_{\mathrm{O}}(\infty)-c_{\mathrm{O}}(0)}=1-\exp \left(-\frac{t}{\tau_{\mathrm{eq}}}\right)
$$

where $\tau_{\text {eq }}$ is the time constant to reach chemical equilibrium. ${ }^{17}$ For a plane sheet one gets $\tau_{\text {eq }}=(h / 2) / K_{\mathrm{O}}$ and for a sphere $\tau_{\text {eq }}=$ $(r / 3) / K_{\mathrm{O}} \cdot{ }^{17}$ Recalling that $K_{\mathrm{O}}$ is a consequence of the boundary condition one concludes

$$
\begin{array}{cc}
\text { Plane sheet: } \quad \tau_{\mathrm{eq}} \equiv t_{\mathrm{c}}^{0}=\frac{c_{\mathrm{O}}(0)}{w(0) \Re_{\mathrm{O}}^{0}} ; \quad K_{\mathrm{O}}^{h \rightarrow 0}=\frac{h}{2} \frac{1}{t_{\mathrm{c}}^{0}}=\frac{h}{2} w(0) \frac{\Re_{\mathrm{O}}^{0}}{c_{\mathrm{O}}(0)} \\
\text { Sphere: } \quad \tau_{\mathrm{eq}} \equiv t_{\mathrm{c}}^{0}=\frac{c_{\mathrm{O}}(0)}{w(0) \Re_{\mathrm{O}}^{0}} ; \quad K_{\mathrm{O}}^{r \rightarrow 0}=\frac{r}{3} \frac{1}{t_{\mathrm{c}}^{0}}=\frac{r}{3} w(0) \frac{\Re_{\mathrm{O}}^{0}}{c_{\mathrm{O}}(0)}
\end{array}
$$

Comparing $K_{\mathrm{O}}^{h \rightarrow \infty}$ for a thick plane sheet in eqn (15) with $K_{\mathrm{O}}^{h, r \rightarrow 0}$ for an extremely thin plane sheet or for powder samples, respectively, shows that, in the latter case, the oxygen exchange coefficient becomes independent of $D_{\mathrm{O}}$. This means that the oxygen exchange rate $\mathfrak{R}_{\mathrm{O}}^{0}$ can be directly determined from the time constant $\tau_{\text {eq }}$ in eqn (20), e.g. in an isothermal isotope exchange (IIE) experiment ${ }^{13}$ or in a thermogravimetric relaxation experiment ${ }^{17}$ carried out in the surface exchange regime. Further, eqn (21) suggests a direct experimental check of the postulate in eqn (14): if the postulate is valid the time constant $\tau_{\text {eq }}$ should not depend on the surface-tovolume ratio of the sample in the surface exchange regime.

The arguments displayed above offer a clue to explain the differences in the reported range of apparent activation energies (s. Armstrong et al., ${ }^{13}$ Table 5). Armstrong et al. ${ }^{13}$ determined $K_{\mathrm{O}}$ values via IIE clearly in the surface exchange regime (spheres: $200-300 \mathrm{~nm}, w_{\mathrm{O}}(0)=1$ ) according to eqn (21)

$$
\text { Surface exchange regime: } \quad K_{\mathrm{O}}^{r \rightarrow 0}=\frac{r}{3} \frac{\Re_{\mathrm{O}}^{0}}{c_{\mathrm{O}}(0)}
$$

Other studies (s. references compiled in Table 5 of Armstrong et $a l .{ }^{13}$ ) which determined $K_{\mathrm{O}}$ values via IEDP/SIMS or ECR, respectively, as well as PIE-based ${ }^{9-11}$ and GPE/GPA-based ${ }^{4,14,16}$ investigations often remained in the diffusion controlled regime $\left(h \gg l_{\mathrm{c}}^{0}\right)$ and therefore unintentionally tended to determine a parameter corresponding to the following expression for the surface exchange coefficient

Diffusion controlled regime:

$$
K_{\mathrm{O}}^{h \rightarrow \infty}=\left(w_{\mathrm{O}}(0) \cdot D_{\mathrm{O}} \cdot \frac{\Re_{\mathrm{O}}^{0}}{c_{\mathrm{O}}(0)}\right)^{1 / 2}
$$

The totally different temperature dependencies which are expected (activation energy for $\mathfrak{R}_{\mathrm{O}}^{0}$ only in eqn (22) and the mean value of the activation energy for $\mathfrak{R}_{\mathrm{O}}^{0}$ and for $D_{\mathrm{O}}$ in eqn (23)) comprise the reported range of apparent activation energies for the two entirely different experimental scenarios and further supports the postulate in eqn (14). Eqn (23) obviously allows to rationalise the $K_{\mathrm{O}} \propto D_{\mathrm{O}}{ }^{1 / 2}$ correlation reported in the literature. ${ }^{1}$

In the following section it is demonstrated how the oxygen exchange rate $\mathfrak{R}_{\mathrm{O}}^{0}$ can also be measured via an ${ }^{18} \mathrm{O}$ exchange experiment on a thick plate-like sample with SIMS depth profiling, that is, in an experimental situation where the oxygen exchange is clearly in the diffusion controlled regime, $h \gg l_{\mathrm{c}}^{0}$. In 2001 Fielitz and Borchardt, ${ }^{23}$ without, however, discussing the physical meaning of $\tau_{\mathrm{eq}}{ }^{*}$, pointed out that the corresponding solution of the diffusion equation as given by $\mathrm{Crank}^{20}$ should be used in the following form

$$
\begin{aligned}
& \frac{c(x, t)-c_{\infty}}{c_{\mathrm{g}}-c_{\infty}} \\
& =\operatorname{erfc}\left(\frac{x}{\sigma^{*}}\right)-\exp \left(2 \frac{x}{\sigma^{*}} \sqrt{\frac{t}{\tau_{\mathrm{eq}}{ }^{*}}}+\frac{t}{\tau_{\mathrm{eq}}{ }^{*}}\right) \operatorname{erfc}\left(\frac{x}{\sigma^{*}}+\sqrt{\frac{t}{\tau_{\mathrm{eq}}{ }^{*}}}\right) \\
& \text { with } \sigma^{*} \equiv 2 \sqrt{D_{\mathrm{O}}{ }^{*} t} \text { and } \tau_{\mathrm{eq}}{ }^{*} \equiv \frac{D_{\mathrm{O}}{ }^{*}}{\left(K_{\mathrm{O}^{*}}\right)^{2}}
\end{aligned}
$$


to fit SIMS depth profiles (s. elsewhere ${ }^{23}$ for further details) in order to extract the tracer isotope diffusion length $\sigma^{*}$ and the time constant $\tau_{\mathrm{eq}}{ }^{*}$ which is the time necessary to obtain isotope equilibrium at the surface $(x=0)$. As the boundary condition eqn (8) was used to solve the diffusion equation ${ }^{20}$ one can use the set of eqn (19) (with $w_{\mathrm{O}}(0)=1$ for an isotope exchange experiment)

$$
\begin{aligned}
& t_{\mathrm{c}}^{0 *}=\tau_{\mathrm{eq}}{ }^{*}=\frac{c_{\mathrm{O}}(0)}{\Re_{\mathrm{O}}^{0}} ; \quad l_{\mathrm{c}}^{0 *}=\sqrt{D_{\mathrm{O}}{ }^{*} \cdot \tau_{\mathrm{eq}}} ; \\
& K_{\mathrm{O}}^{*}=\sqrt{\frac{D_{\mathrm{O}}{ }^{*}}{\tau_{\mathrm{eq}}{ }^{*}}}=\sqrt{D_{\mathrm{O}}{ }^{*} \frac{\Re_{\mathrm{O}}^{0}}{c_{\mathrm{O}}(0)}}
\end{aligned}
$$

This means that the oxygen exchange rate $\mathfrak{R}_{\mathrm{O}}^{0}$ can be directly determined by the time constant $\tau_{\text {eq }}{ }^{*}$ in eqn (24). It should be underlined that the specific form of the solution Fielitz and Borchardt ${ }^{23}$ proposed explicitly contains the function $K_{\mathrm{O}}{ }^{*}=$ $\sqrt{D_{\mathrm{O}}{ }^{*} / \tau_{\mathrm{eq}}}{ }^{*}$ (compare to $\tau_{\mathrm{eq}}{ }^{*}$ in eqn (24)), whereas this fact is not evident in the original form given by Crank. ${ }^{20}$

The presented revision of the principal nature of the surface exchange coefficient has been essentially triggered by the publications of Armstrong et al. ${ }^{12,13}$ since these investigations demonstrated clearly that the experimentally determined apparent activation energies for $K_{\mathrm{O}}{ }^{*}$ strongly depend on the kinetic regime of the respective experiments. Armstrong et $a .^{12,13}$ studied a relatively broad spectrum of materials (various perovskite MIECs like LC, LF, LSM, LSF, LSC or LSCF as well as two widely used oxygen electrolyte materials, namely YSZ and GDC). The similarities of the regime dependency of $K_{\mathrm{O}}{ }^{*}$ therefore suggests that their observation does not reflect a specific property of a class of materials, but rather an underlying universal phenomenon. It should, however, be recalled that there is another method to determine $K_{\mathrm{O}}{ }^{*}$ on powders which does not find any dependence of the apparent activation energies on the kinetic regime. Bouwmeester et al. ${ }^{9}$ and Yoo et al. ${ }^{10,11}$ used a novel pulse ${ }^{18} \mathrm{O}-{ }^{16} \mathrm{O}$ isotopic exchange (PIE) technique for the rapid determination of the oxygen surface exchange rate of oxide ion conductors. For this method powders with an average particle size in the range $0.4-5.0 \mu \mathrm{m}$ are used. The reported apparent activation energies did not significantly differ from the values determined on bulk samples via IEDP/SIMS (or ECR, respectively). Recent GPE/GPA-based work $^{4,14,16}$ comprises similar activation energies for the surface exchange coefficient and for the oxygen diffusivity.

During the PIE experiment, a powder sample is loaded in a packed-bed micro-reactor. Maintaining conditions of chemical equilibrium, the response to an ${ }^{18} \mathrm{O}$-enriched pulse fed through the reactor under continuous flow conditions is measured by mass spectrometric analysis of the gas phase fractions of oxygen isotopomers $\left({ }^{18} \mathrm{O}_{2},{ }^{16} \mathrm{O}^{18} \mathrm{O}\right.$ and $\left.{ }^{16} \mathrm{O}_{2}\right)$ at the exit of the reactor. The essential difference between $\mathrm{PIE}^{9-11}$ and $\mathrm{IIE}^{12,13}$ is the residence time between the ${ }^{18} \mathrm{O}_{2}$ atmosphere and the powder sample: for PIE the mean residence time is 5 to $40 \mathrm{~ms},{ }^{9-11}$ whereas in an IIE experiment the residence time is 15 to $40 \mathrm{~min} \cdot{ }^{12,13} \mathrm{In}$ this context, it is worth recalling that the surface exchange
Table 1 Characteristic dimensionless parameters of the kinetic regimes

\begin{tabular}{llll}
\hline Kinetic regime & $L=(h / 2)\left(K_{\mathrm{O}} / D_{\mathrm{O}}\right)$ & $\phi\left(\beta_{1}, L\right)$ & $\beta_{1}{ }^{2}$ \\
\hline Surface control & $<0.3$ & 1 & $L$ \\
Mixed regime & 0.3 to 30 & - & $-\overline{\pi^{2}} / 4$ \\
Diffusion regime & $>30$ & $8 / \pi^{2}$ &
\end{tabular}

regime is not reached before the ${ }^{18} \mathrm{O}$ concentration gradient (in the bulk of a powder particle) practically disappears, which means that the value of $D_{\mathrm{O}}{ }^{*}$ has become no longer relevant for the ${ }^{18} \mathrm{O}$ exchange process. Since principally a certain (finite) diffusion time $t$ has to elapse before an ${ }^{18} \mathrm{O}$ concentration gradient in a particle with nominal radius $r$ disappears, IIE seems to be more suitable than PIE to operate in a true surface exchange regime. This can be easily demonstrated by a straightforward estimation: the condition $\sqrt{D_{\mathrm{O}}{ }^{*} t}>r$ relates the necessary diffusion length, which is required to reach the surface exchange regime, to the particle radius. Estimating an average particle radius $r_{\mathrm{PIE}} \approx 3 \mu \mathrm{m}^{9-11}$ for the PIE experiment and an average particle radius $r_{\text {IIE }} \approx 0.3 \mu \mathrm{m}^{12,13}$ for the IIE experiment one has $t_{\mathrm{PIE}} / t_{\mathrm{IIE}}=\left(r_{\mathrm{PIE}} / r_{\mathrm{IIE}}\right)^{2} \approx 100$ as a condition to reach the surface exchange regime in the published PIE studies compared to the published IIE studies. Since, however, the experimentally realized residence times $t_{\mathrm{PIE}}$ during the PIE experiment are only in the millisecond range, ${ }^{9-11}$ so that one can estimate the ratio $t_{\mathrm{PIE}} / t_{\mathrm{IIE}} \approx 40 \mathrm{~ms} / 40 \mathrm{~min}=1.7 \times 10^{-5}$, it cannot be excluded that PIE operates in a transitory, i.e. mixed controlled regime, similarly to the great majority of the published IEDP/SIMS studies.

Likewise the GPE/GPA-based investigations ${ }^{4,14,16}$ use macroscopic samples and fairly long annealing times (for details see Appendix B). This inevitably implies a shift to $L$ values typical for a mixed controlled or diffusion controlled regime (see Table 1 and Appendix C).

\section{Summary}

In 1998 Maier $^{6}$ proposed eqn (2) according to which the exchange rate $\mathfrak{R}_{\mathrm{O}}^{0}$ (for oxygen) is proportional to the surface exchange coefficient $K_{\mathrm{O}}$. Since then two well documented significant experimental findings became known which contradicted the general validity of eqn (2):

(i) Instead of the theoretically expected relation ${ }^{15} K_{\mathrm{O}}{ }^{*} / D_{\mathrm{O}}{ }^{*} \cong$ $\tilde{K}_{\mathrm{O}} / \tilde{D}_{\mathrm{O}}$ the experiments yield ${ }^{7} K_{\mathrm{O}}{ }^{*} / D_{\mathrm{O}}{ }^{*}>\tilde{K}_{\mathrm{O}} / \tilde{D}_{\mathrm{O}}$.

(ii) If measured in the surface exchange regime $\left(h \ll l_{\mathrm{c}}^{0}\right)$ the apparent activation energies for $K_{\mathrm{O}}{ }^{*}$ and $\tilde{K}_{\mathrm{O}}$ are very small ${ }^{13,17}$ whereas the apparent activation energies measured in the mixed controlled regime $\left(h \approx l_{\mathrm{c}}^{0}\right.$ ) or in the diffusion controlled regime $\left(h \gg l_{\mathrm{c}}^{0}\right)$ are considerably larger (s. Armstrong et al., ${ }^{13}$ Table 5).

These two surprising experimental observations, but especially the latter one, raise doubtful questions about the general validity of eqn (2), which necessitate a thorough fundamental rediscussion of the surface exchange coefficient for oxygen (on oxide surfaces). 
Following principally Maier's ${ }^{6}$ procedure the simplest possible experimental situation was chosen, i.e. an oxide surface close to chemical equilibrium (s. eqn (6)). For a complete picture, one has, however, to include the diffusive transport of oxygen (s. eqn (7)) and the fact that at the gas/solid interface there is a boundary condition to be respected. For the gas/solid interface of a homogeneous semi-infinite oxide this is eqn (8). This boundary condition inevitably leads to a mathematical correlation between $K_{\mathrm{O}}$ and $D_{\mathrm{O}}$ (s. eqn (11)), which, at first glance, seems to be only a formal recipe to calculate the so-called characteristic length $l_{\mathrm{c}}^{0}$. Through the definition of a characteristic time $t_{\mathrm{c}}^{0}$, this parameter can be attributed a physical meaning in eqn (13) as $t_{\mathrm{c}}^{0}$ is the time necessary for the relaxation of a small change of the chemical potential of oxygen at the surface, $\delta \mu_{\mathrm{O}}(0)$. Further, it becomes obvious that in eqn (2) the term $K_{\mathrm{O}} / \Delta x$ (as formulated by Maier ${ }^{6}$ ) is equivalent to the inverse characteristic time $t_{\mathrm{c}}^{0}$. It is therefore consistent to correlate the oxygen exchange rate $\mathfrak{R}_{\mathrm{O}}^{0}$ and the characteristic time $t_{\mathrm{c}}^{0}$ directly, as postulated through eqn (14). This postulate and the boundary condition (eqn (8)) entail an explicit dependency between the (surface) exchange rate and the (bulk) diffusivity of oxygen $K_{\mathrm{O}}=$ $K_{\mathrm{O}}\left(D_{\mathrm{O}}, \Re_{\mathrm{O}}^{0}\right.$ ) (s. eqn (15)). At the same time such a dependency implies that for more complicated diffusion boundary conditions more complicated functions for $K_{\mathrm{O}}$ have to be expected.

The relations for $K_{\mathrm{O}}$ in the different kinetic regimes derived in the framework of the proposed novel interpretation of the standard theoretical concept ${ }^{6}$ for $K_{\mathrm{O}}$ finally enable to unambiguously explain the two experimental observations (i) and (ii). In the presented model eqn (2) is only valid for the special case of the true surface exchange regime (s. eqn (21)). The parameter $\Delta x$ in eqn (2), however, then is no longer a constant, but corresponds to the surface-to-volume ratio of the sample. As a consequence, the time constant $\tau_{\mathrm{eq}}$ to reach equilibrium (s. eqn (20) and (21)) should not depend on the surface-tovolume ratio of the sample in the surface exchange regime.

\section{Appendix A}

For a thin surface layer $\delta x^{\prime}(x=0)$ the following expression can be formulated

$$
\delta x^{\prime}(0)=\frac{\delta x(0)}{w_{\mathrm{O}}(0)}=\frac{l_{\mathrm{c}}^{0}}{w_{\mathrm{O}}(0)}
$$

If $\delta x^{\prime}(0)$ is sufficiently small and/or if the oxygen flux $j_{\mathrm{O}}^{K}(0)$ is approximately constant within the layer of thickness $\delta x^{\prime}(0)$ one obtains with eqn (6)

$$
\frac{\mathrm{d}}{\mathrm{d} t} c_{\mathrm{O}}(0)=\frac{j_{\mathrm{O}}^{K}(0)}{\delta x^{\prime}(0)}=-\frac{\Lambda_{\mathrm{O}}}{\delta x^{\prime}(0)} \delta \mu_{\mathrm{O}}(0)=\left.\frac{\partial c_{\mathrm{O}}}{\partial \mu_{\mathrm{O}}}\right|_{x=0} \frac{\mathrm{d}}{\mathrm{d} t} \delta \mu_{\mathrm{O}}(0)
$$

That is, the time constant $t_{\mathrm{c}}^{0}$ for the exponential relaxation kinetics of a small oxygen potential change $\delta \mu_{\mathrm{O}}(0)$ at the surface is given by

$$
\frac{1}{t_{\mathrm{c}}^{0}}=\left.\frac{\Lambda_{\mathrm{O}}}{\delta x^{\prime}(0)} \frac{\partial \mu_{\mathrm{O}}}{\partial c_{\mathrm{O}}}\right|_{x=0}
$$

Together with $K_{\mathrm{O}}=\Lambda_{\mathrm{O}} \partial \mu_{\mathrm{O}} /\left.\partial c_{\mathrm{O}}\right|_{x=0}$ and eqn (A1), (A3) yields eqn (12).

\section{Appendix B}

A widely applicable reaction model comprises the following consecutive steps: ${ }^{7}$

(1) adsorption dissociation (eq.)

$$
\begin{aligned}
& \frac{1}{2} \mathrm{O}_{2}(\mathrm{~g}) \leftrightarrow \mathrm{O}_{\mathrm{ad}}, \quad K_{1}(T) ; \\
& \Re_{1}=\vec{R}_{1}-\overleftarrow{R}_{1}=\vec{k}_{1} p_{\mathrm{O}_{2}}{ }^{1 / 2}-\overleftarrow{k}_{1}\left[\mathrm{O}_{\mathrm{ad}}\right]
\end{aligned}
$$

(2) charge transfer (rds)

$$
\begin{aligned}
& \mathrm{O}_{\mathrm{ad}}+\mathrm{e}^{\prime} \leftrightarrow \mathrm{O}_{\mathrm{ad}}^{\prime}, \quad K_{2}(T) ; \\
& \Re_{2}=\vec{R}_{2}-\overleftarrow{R}_{2}=\vec{k}_{2}\left[\mathrm{O}_{\mathrm{ad}}\right]\left[\mathrm{e}^{\prime}\right]-\overleftarrow{k}_{2}\left[\mathrm{O}_{\mathrm{ad}}^{\prime}\right]
\end{aligned}
$$

(3) incorporation (eq.):

$$
\begin{aligned}
& \mathrm{O}_{\mathrm{ad}}^{\prime}+\mathrm{V}_{\mathrm{O}}^{\bullet \bullet} \leftrightarrow \mathrm{O}_{\mathrm{O}}^{\times}+\mathrm{h}^{\bullet}, \quad K_{3}(T) ; \\
& \Re_{3}=\vec{R}_{3}-\overleftarrow{R}_{3}=\vec{k}_{3}\left[\mathrm{O}_{\mathrm{ad}}^{\prime}\right]\left[\mathrm{V}_{\mathrm{O}}^{\bullet \bullet}\right]-\overleftarrow{k}_{3}\left[\mathrm{O}_{\mathrm{O}}^{\times}\right]\left[\mathrm{h}^{\bullet}\right]
\end{aligned}
$$

The reactions are formulated according to the Kröger-Vink notation, using square brackets for the respective concentrations, $K_{n}$ for the mass action constants $(n=1,2,3), \vec{k}_{n}, \overleftarrow{k}_{n}$ for the forward/ backward rate constants, $\vec{R}_{n}, \overleftarrow{R}_{n}$ for the forward/backward reaction rates and $\mathfrak{R}_{n}$ for the net reaction rates of these reactions. The so called apparent or effective equilibrium exchange rate $\mathfrak{R}_{\mathrm{O}}^{0}$ of the rate determining step (charge transfer (B2) is often assumed as the rate determining step $^{7}$ ) is defined by

$$
\Re_{\mathrm{O}}^{0}=\sqrt{\overleftarrow{R}_{2} \times \vec{R}_{2}}=\left(\overleftarrow{k}_{2}\left[\mathrm{O}_{\mathrm{ad}}^{\prime}\right] \times \vec{k}_{2}\left[\mathrm{O}_{\mathrm{ad}}\right]\left[\mathrm{e}^{\prime}\right]\right)^{1 / 2}
$$

After inserting the mass action constants

$$
K_{1}=K_{\mathrm{ad}}=\frac{\overrightarrow{k_{1}}}{\overleftarrow{k}_{1}}=\frac{\left[\mathrm{O}_{\mathrm{ad}}\right]}{p_{\mathrm{O}_{2}}{ }^{1 / 2}} ; \quad K_{3}=K_{\mathrm{inc}}=\frac{\vec{k}_{3}}{\overleftarrow{k}_{3}}=\frac{\left[\mathrm{O}_{\mathrm{O}}^{\times}\right]\left[\mathrm{h}^{\bullet}\right]}{\left[\mathrm{O}_{\mathrm{ad}}^{\prime}\right]\left[\mathrm{V}_{\mathrm{O}}^{\bullet}\right]}
$$

into eqn (B4) and defining notational abbreviations $c_{\mathrm{e}} \equiv\left[\mathrm{e}^{\prime}\right], c_{\mathrm{h}} \equiv$ $\left[\mathrm{h}^{\bullet}\right], c_{\mathrm{v}} \equiv\left[\mathrm{V}_{\mathrm{O}}^{\bullet \bullet}\right], c_{\mathrm{O}} \equiv\left[\mathrm{O}_{\mathrm{O}}^{\times}\right]$one finally gets if, e.g., charge transfer is rate determining

$$
\Re_{\mathrm{O}}^{0}=\left(\frac{\overleftarrow{k}_{2} \vec{k}_{2} K_{\mathrm{ad}}}{K_{\text {inc }}}\right)^{1 / 2}\left(c_{\mathrm{O}} c_{\mathrm{e}} c_{\mathrm{h}}\right)^{1 / 2} \frac{p_{\mathrm{O}_{2}}{ }^{1 / 4}}{c_{\mathrm{v}}{ }^{1 / 2}}
$$

Eqn (B6) demonstrates in a very convincing manner that the surface exchange coefficient will have a non-trivial temperature dependence in the surface controlled regime $\left(K_{\mathrm{O}} \propto \mathfrak{R}_{\mathrm{O}}^{0}\right.$, see eqn (21)). The resulting apparent activation energy should generally be less than the average energy of $D_{\mathrm{O}}$ and $\mathfrak{R}_{\mathrm{O}}^{0}$. In the diffusion controlled regime $\left(K_{\mathrm{O}} \propto \sqrt{D_{\mathrm{O}} \Re_{\mathrm{O}}^{0}}\right.$, see eqn (15)) the resulting apparent activation energy is the average activation energy of $D_{\mathrm{O}}$ and $\mathfrak{R}_{\mathrm{O}}^{0}$.

We will now demonstrate that there is an equivalence of the definitions of the phenomenological parameters used in 
Maier's approach ${ }^{6}$ and the approach of Klier and Kučera. ${ }^{24}$ Comparing both approaches it becomes evident that recent work, ${ }^{4,14}$ based on the work of Klier and Kučera, ${ }^{24}$ uses the same phenomenological description for their consecutive model as Maier ${ }^{6}$ and the present authors (neglecting, however, the charge transfer step, which seems to be a weakness of this approach). With $r_{\mathrm{H}}$ for the "heterogeneous" reaction rate, $r_{\mathrm{a}}$ for the (dissociative) "adsorption" rate, and $r_{\mathrm{i}}$ for the "incorporation" rate they obtain (see eqn (8) and (12) in Farlenkov et al. ${ }^{14}$ ) $r_{\mathrm{H}}=r_{\mathrm{a}} \cdot r_{\mathrm{i}} /\left(r_{\mathrm{a}}+r_{\mathrm{i}}\right)$, i.e. they set $1 / r_{\mathrm{H}}=1 / r_{\mathrm{a}}+1 / r_{\mathrm{i}}$ as expected for a consecutive model. In Maier's notation this would become $1 / \mathfrak{R}^{0}=1 / \mathfrak{R}_{1}+1 / \mathfrak{R}_{3}$ if the charge transfer step (eqn (B2)) is neglected (in order to facilitate the comparison). In eqn (13) of Farlenkov et al. ${ }^{14}$ the surface exchange coefficient, $k$, without specifying the kinetic regime, is defined as $k=$ const. $\times r_{\mathrm{H}}$ (where the constant contains the specific data of the material, which have only a negligible temperature dependence). This corresponds formally to the relation originally proposed by Maier $^{6}$ as well as to our result for the mere surface controlled regime, $K_{\mathrm{O}}=$ const. $\times \mathfrak{R}_{\mathrm{O}}^{0}$, as expressed by eqn (21). It is, however, debatable (see Appendix C) whether the pure surface controlled regime can anyhow be studied if the dense (polycrystalline and single crystalline) samples have a thickness of $5 \mathrm{~mm}$ and undergo annealing times of more than $5 \times 10^{4} \mathrm{s.}^{14}$

\section{Appendix C}

The most direct way to follow the kinetics of oxygen uptake or release in situ at high temperatures is a gravimetric experiment. ${ }^{17}$ For plate-like samples with thickness $h$ the mass change, $\Delta m(t)$, if normalized to the mass change at equilibrium, $\Delta m\left(t_{\mathrm{eq}}\right)$, is given by $\left(\mathrm{Crank}^{20}\right.$ p. 60)

$$
\begin{gathered}
\frac{\Delta m(t)}{\Delta m\left(t_{\mathrm{eq}}\right)}=1-\sum_{n=1}^{\infty} \frac{2 L^{2}}{\beta_{n}{ }^{2}\left(\beta_{n}{ }^{2}+L^{2}+L\right)} \exp \left(-\beta_{n}{ }^{2} \frac{D_{\mathrm{O}} t}{(h / 2)^{2}}\right) \\
\text { with } \quad L \equiv \frac{h}{2} \frac{K_{\mathrm{O}}}{D_{\mathrm{O}}}
\end{gathered}
$$

The dimensionless quantities $\beta_{n}$ are positive roots of $\beta_{n} \tan \beta_{n}=$ $L$. As the infinite sum in eqn (C1) converges rapidly one has approximately $^{17}$

$$
\begin{aligned}
\frac{\Delta m(t)}{\Delta m\left(t_{\mathrm{eq}}\right)} & \approx 1-\frac{2 L^{2}}{\beta_{1}^{2}\left(\beta_{1}^{2}+L^{2}+L\right)} \exp \left(-\beta_{1}^{2} \frac{D_{\mathrm{O}} t}{(h / 2)^{2}}\right) \\
& =1-\phi\left(\beta_{1}, L\right) \exp \left(-\beta_{1}{ }^{2} \frac{D_{\mathrm{O}} t}{(h / 2)^{2}}\right)
\end{aligned}
$$

where $D_{\mathrm{O}}$ is the effective oxygen diffusion coefficient in the solid and $K_{\mathrm{O}}$ is the oxygen surface exchange coefficient defined by eqn (1). Positive roots of $\beta_{1} \tan \beta_{1}=L$ are plotted in Fig. 2 which allows a graphical visualisation of the kinetic regimes. The values of the resulting characteristic dimensionless parameters of the kinetic regimes are compiled in Table 1.

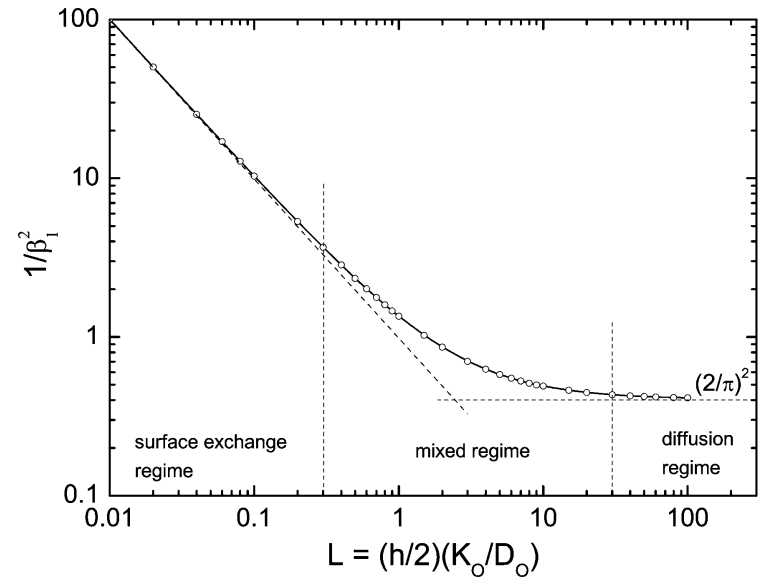

Fig. 2 Graphical visualisation of the kinetic regimes ${ }^{17}$ (open points are positive roots of $\beta_{1} \tan \beta_{1}=L$ tabulated by Carslaw and Jaeger ${ }^{25}$ ). The diffusion regime is characterised by $\beta_{1}^{2} \simeq \pi^{2} / 4$ and the surface exchange regime by $\beta_{1}^{2} \simeq L$.

\section{Acknowledgements}

We are indebted to Martin Schmücker, Aldo Steinfeld, Robert Pitz-Paal, Clemens Feldmann and their co-workers for valuable discussions. Financial support by the Initiative and Networking Fund of the Helmholtz Association of German Research Centers within the Virtual Institute SolarSynGas as well as by Deutsche Forschungsgemeinschaft (DFG) for one of the authors (P. F.) is gratefully acknowledged.

\section{References}

1 J. A. Kilner, R. A. De Souza and I. C. Fullarton, Solid State Ionics, 1996, 86-88, 703.

2 J. A. Kilner, S. J. Skinner and H. H. Brongersma, J. Solid State Electrochem., 2011, 15, 861.

3 J. Blair and D. S. Mebane, Solid State Ionics, 2015, 270, 47.

4 M. V. Ananyev, E. S. Tropin, V. A. Eremin, A. S. Farlenkov, A. S. Smirnov, A. A. Kolchugin, N. M. Porotnikova, A. V. Khodimchuk, A. V. Berenov and E. Kh. Kurumchin, Phys. Chem. Chem. Phys., 2016, 18, 9102.

5 R. J. Chater, S. Carter, J. A. Kilner and B. C. H. Steele, Solid State Ionics, 1992, 53-56, 859.

6 J. Maier, Solid State Ionics, 1998, 112, 197.

7 R. A. De Souza, Phys. Chem. Chem. Phys., 2006, 8, 890.

8 R. Merkle and J. Maier, Angew. Chem., Int. Ed., 2008, 47, 3874.

9 H. J. M. Bouwmeester, C. Song, J. Zhu, J. Yi, M. van Sint Annaland and B. A. Boukamp, Phys. Chem. Chem. Phys., 2009, 11, 9640.

10 C.-Y. Yoo, B. A. Boukamp and H. J. M. Bouwmeester, J. Solid State Electrochem., 2011, 15, 231.

11 C.-Y. Yoo and H. J. M. Bouwmeester, Phys. Chem. Chem. Phys., 2012, 14, 11759.

12 E. N. Armstrong, K. L. Duncan, D. J. Oh, J. F. Weaver and E. D. Wachsman, J. Electrochem. Soc., 2011, 158, B283.

13 E. N. Armstrong, K. L. Duncan and E. D. Wachsman, Phys. Chem. Chem. Phys., 2013, 15, 2298. 
14 A. S. Farlenkov, M. V. Ananyev, V. A. Eremin, N. M. Porotnikova, E. K. Kurumchin and B.-T. Melekh, Solid State Ionics, 2016, 290, 108.

15 J. Maier, Solid State Ionics, 2000, 135, 575.

16 A. Berenov, A. Atkinson, J. Kilner, M. Ananyev, V. Eremin, N. Porotnikova, A. Farlenkov, E. Kurumchin, H. J. M. Bouwmeester, E. Bucher and W. Sitte, Solid State Ionics, 2014, 268, 102.

17 N. Knoblauch, L. Dörrer, P. Fielitz, M. Schmücker and G. Borchardt, Phys. Chem. Chem. Phys., 2015, 17, 5849.

18 S. Ackermann, J. R. Scheffe and A. Steinfeld, J. Phys. Chem. C, 2014, 118, 5216.
19 H. Schmalzried, Solid State Reactions, Verlag Chemie, Weinheim, 1974, p. 54.

20 J. Crank, The Mathematics of Diffusion, Oxford University Press, 2nd edn, 1975, p. 36.

21 J. Claus, M. Leonhardt and J. Maier, J. Phys. Chem. Solids, 2000, 61, 1199.

22 M. Leonhardt, R. A. De Souza, J. Claus and J. Maier, J. Electrochem. Soc., 2002, 149, J19.

23 P. Fielitz and G. Borchardt, Solid State Ionics, 2001, 144, 71. 24 K. Klier and E. Kučera, J. Phys. Chem. Solids, 1966, 27, 1087. 25 H. S. Carslaw and J. C. Jaeger, Conduction of Heat in Solids, Clarendon Press, 2nd edn, 1959. 